\title{
Clinical Pharmacokinetics and Pharmacodynamics of Dalcetrapib
}

\author{
Donald M. Black ${ }^{1} \cdot$ Darren Bentley $^{2} \cdot$ Sunny Chapel $^{3} \cdot$ Jongtae Lee $^{3} \cdot$ Emily Briggs $^{3} \cdot$ \\ Therese Heinonen ${ }^{1}$
}

Published online: 5 May 2018

(C) The Author(s) 2018

\begin{abstract}
The cholesterol ester transfer protein (CETP) inhibitor dalcetrapib has been under evaluation for its potential to prevent cardiovascular (CV) events for almost two decades. The current clinical development program, representing new advances in precision medicine and focused on a genetically defined population with acute coronary syndrome (ACS), is supported by a large body of pharmacokinetic and pharmacodynamic data as well as substantial clinical experience in over 13,000 patients and volunteers. Dalcetrapib treatment of $600 \mathrm{mg} /$ day produces significant inhibition of CETP activity, and has been utilized in phase II and III studies, including CV endpoint trials. Numerous studies have investigated the interactions between dalcetrapib and most drugs commonly prescribed to $\mathrm{CV}$ patients and have not demonstrated any clinically significant effects. Evaluations in patients with renal and hepatic impairment demonstrate a greater exposure to dalcetrapib than in the non-impaired population, but longterm clinical studies including patients with mild to moderate hepatic and renal dysfunction demonstrate no increase in adverse events. Safety pharmacology and toxicology studies as well as the clinical safety experience support the continuing development of dalcetrapib as an adjunct to 'standard of care' for the ACS population. This article provides a full review of the pharmacokinetics, as well as pharmacodynamics and pharmacology, of dalcetrapib in the context of a large clinical program.
\end{abstract}

Donald M. Black

Dblack@dalcorpharma.com

1 Dalcor Pharmaceuticals UK Limited, 123 Wellington Road South, Stockport, Cheshire SK1 3TH, UK

2 F. Hoffmann-LaRoche Ltd, Basel, Switzerland

3 A2PG, Ann Arbor, Michigan, USA

\section{Key Points}

Dalcetrapib is a selective cholesterol ester transfer protein (CETP) inhibitor that modulates reverse cholesterol transport. An analysis of a large cardiovascular (CV) endpoint study has indicated that dalcetrapib reduces $\mathrm{CV}$ events in patients with the AA genotype at rs7309 of the adenylate cyclase type 9 (ADCY9) gene.

The oral bioavailability of dalcetrapib is low and limited by extensive first-pass metabolism. The timing and size of a meal also influences the concentration and pharmacokinetics are not affected by age, race, weight, ethnicity, or $A D C Y 9$ genotype.

Dalcetrapib has no clinically relevant drug-drug interactions with statins (HMG-CoA reductase inhibitors), ezetimibe, or other drugs that are commonly utilized in patients with CV disease.

\section{Introduction}

Dalcetrapib is an inhibitor of cholesterol ester transfer protein (CETP) and is presently under development to reduce cardiovascular (CV) morbidity and mortality in patients at risk of $\mathrm{CV}$ events. Although current therapeutic options have proved effective in reducing morbidity and mortality from $\mathrm{CV}$ disease (CVD), appreciable residual risk for $\mathrm{CV}$ events remains, and CVD is one of the leading causes of death worldwide. 
One approach to reduce this residual risk has been to target high-density lipoprotein (HDL) cholesterol (HDLC). Epidemiology studies show that plasma levels of HDL$\mathrm{C}$ inversely correlate with $\mathrm{CV}$ risk. HDL particles play a central role in trafficking of cholesterol from peripheral tissues to the liver for elimination (reverse cholesterol transport [RCT]) but are also believed to have a number of other anti-atherogenic functions [1]. However, the potential benefits of increasing HDL-C concentrations have not been established despite decades of effort with a series of new chemical entities.

HDL function, as measured by the capacity to accept cholesterol from peripheral tissue, has also been shown to be inversely related to atherosclerotic burden. It is therefore anticipated that therapies aimed at enhancing HDL function will arrest the progression of atherosclerosis and reduce $\mathrm{CV}$ risk. One potential therapeutic strategy for raising HDL-C and increasing HDL functionality is to reduce CETP activity. CETP mediates heterotypic transfer of neutral lipids such as cholesteryl ester (CE) and triglycerides from HDL to apolipoprotein B (apoB)-containing lipoproteins such as low-density lipoprotein (LDL) and very low-density lipoprotein (VLDL). It also facilitates homotypic transfer of CEs among HDL sub-particles and promotes generation of pre- $\beta$-HDL, which participates in the first step of RCT [2].

CETP inhibition has been a therapeutic target since the early 1990s, following the publication of a genetically defined subpopulation in Japan who exhibited abnormally high HDL-C levels as well as longevity and reduced atherosclerosis [3]. In addition, animals that have CETP are more prone to atherosclerosis [1]. CETP mediates the transfer of triglyceride and CE between lipoproteins, and therefore inhibition of the CETP pathway can increase the concentration of HDL-C. The most crucial role for HDL is cholesterol efflux capacity. As measured by recent methods, cholesterol efflux capacity is indicative of changes in atherosclerosis, due to the ability to shift cholesterol from foam cells in atherosclerotic plaques [4]. However, the HDL-C concentration is not predictive of the composition or functionality of HDL [2], and patients who have experienced a myocardial infarction (MI) may have impaired HDL functionality (as measured by efflux or anti-inflammatory properties) as compared with patients who have not had a recent MI [5].

The first CETP inhibitor (CETPi) in large-scale clinical trials was torcetrapib but, due to probable off-target effects, torcetrapib raised blood pressure and increased serum aldosterone, leading to higher CV events and mortality [6]. The results were surprising, but other CETPi drugs were cleared of this off-target effect and entered into full-scale development [7]. Several drugs are very powerful inhibitors of CETP, and affect both heterotypic (e.g., between
HDL and LDL) and homotypic (e.g., between HDL subparticles) CE transfer. These drugs, anacetrapib and evacetrapib, also had significant effects on decreasing LDL cholesterol (LDL-C) concentration as well as increasing HDL-C. Both of these drugs have now completed evaluation in large $\mathrm{CV}$ endpoint studies, and their further development has been terminated [8, 9]. The results of these studies indicate that a general population treated with CETPi may derive a limited benefit in terms of a decrease in the risk of coronary artery diseases.

\section{History of Dalcetrapib}

Dalcetrapib was invented by Japan Tobacco (JTT) and is structurally distinct from other inhibitors of CETP, with a smaller molecular weight and a lack of trifluoride moieties. Unlike the other inhibitors of CETP, dalcetrapib only affects the heterotypic CE transfer, and does not affect LDL-C concentrations or apoB [10]. JTT licensed the drug to Roche, and Roche completed the full characterization of the clinical pharmacology of the drug before embarking on a series of phase II and III studies, and ultimately the CV event study dal-OUTCOMES. The study, which enrolled 15,871 patients onto either placebo or dalcetrapib $600 \mathrm{mg} /$ day, was designed to demonstrate a reduction in $\mathrm{CV}$ events in acute coronary syndrome (ACS) patients on a background of therapy to bring LDL-C concentrations to below $100 \mathrm{mg} / \mathrm{dL}$. However, the study was halted after an average treatment period of 31 months due to futility, and while the drug was well-tolerated, there was no discernible benefit [11].

With the hypothesis that the response to dalcetrapib may vary according to the genetic background, a pharmacogenomic evaluation using a genome-wide approach in the dal-OUTCOMES study (discovery cohort, $n=5749$ ) was then conducted. Of the 5,543,264 common genetic variants analyzed, CV events were associated with the adenylate cyclase type 9 ( $A D C Y 9$ ) gene on chromosome 16. The region includes a single genotyped single nucleotide polymorphism (SNP) that passed the significance threshold $\left(P=2.41 \times 10^{-8}\right)$. The main association between rs1967309 and CV events was robust to further adjustments for baseline hypertension, hypercholesterolemia, diabetes mellitus, smoking status, previous percutaneous coronary intervention, previous coronary artery bypass graft, and MI prior to the index event. There was no detectable genetic effect for rs1967309 in the placebo arm alone [12]. Results demonstrated that patients who were homozygotes for the minor allele (AA) at variant rs1967309 in the ADCY9 gene experienced a $39 \%$ reduction in the pre-specified composite endpoint of coronary heart disease (CHD) death, resuscitated cardiac arrest, non-fatal MI, non-fatal ischemic 
stroke, unstable angina, or unanticipated coronary revascularization with dalcetrapib compared with placebo. Patients homozygous for the genotype GG at rs1967309 and treated with dalcetrapib had a $27 \%$ increase in $\mathrm{CV}$ events versus placebo. The results were confirmed through a targeted genotyping panel in the dal-PLAQUE-2 imaging trial that demonstrated a significant reduction of carotid intima-media thickness (representing atherosclerosis regression) in patients with the AA allele treated with dalcetrapib as compared with placebo. Thus, treatment with dalcetrapib in patients with the AA genotype at variant rs1967309 in the $A D C Y 9$ gene appears to confer significant $\mathrm{CV}$ benefit.

Roche subsequently licensed the project to the Swiss branch of DalCor Pharma UK Ltd. Further studies of the effect of dalcetrapib on inflammatory markers such as high-sensitivity $\mathrm{C}$-reactive protein have also correlated with the benefits seen in the AA genotype patients, while improved cholesterol efflux supports the role of CETP inhibition as causative [13]. In an effort to confirm these findings, a prospective $\mathrm{CV}$ outcomes study was initiated (DAL-301): the dal-GenE study (NCT02525939) [46]. The goal is to reduce the risk of recurrent $\mathrm{CV}$ events within a genetically defined population of patients with ACS at a daily dose of two dalcetrapib $300 \mathrm{mg}$ tablets taken once a day.

\section{Absorption, Distribution, Metabolism, Elimination}

Preclinical investigations included an extensive program of drug metabolism, pharmacokinetics, safety pharmacology, and toxicology studies in vitro and in a variety of rodent, leporid, canine, and primate species. Safety pharmacology and toxicology studies did not show any findings that would raise significant concerns about the use of the proposed clinical dose in humans.

Oral bioavailability of dalcetrapib appears to be both dose- and time-dependent. Dalcetrapib active form exposure did not increase in a dose-proportional manner across the dose range tested and exposures at steady state tended to be lower than predicted from single-dose data. The causes of the dose and time dependency have not been identified. Time of drug dosing during the day had no effect on dalcetrapib active form bioavailability.

Dalcetrapib is a thioester (thiophenolester) prodrug, which is rapidly hydrolyzed to generate a pharmacologically active thiol (thiophenol) [10, 14]. The thioester is not systemically available [15-17]. All reported pharmacokinetic data are based on a bioanalytical assay to measure concentrations of the thiol in free, bound, or dimeric form (collectively referred to as 'active form') rather than dalcetrapib [18]. The same basic bioanalytical assay has been used to measure dalcetrapib active form concentrations throughout the clinical program.

The thioester is a lipophilic compound (log $\mathrm{P} 7.0)$ that has low solubility in water and other aqueous media [18]. Aqueous solubility is increased by the presence of surfactants or complexing agents, leading to a higher solubility in human gastrointestinal fluids. However, in most situations the thioester only made up a small proportion of drugrelated material in solution in gastrointestinal fluids. Although the thioester was relatively stable in saliva and fasted gastric fluid, thioester hydrolysis was rapid in simulated gastrointestinal fluids and in the presence of pancreatin or lipase: in intestinal fluids, the thioester had a half-life $\left(t_{1 / 2}\right)$ of a few seconds. Based on these data it is anticipated that when administered with food the thioester will be rapidly hydrolyzed to the thiol as it is solubilized. This is further supported by observations from preclinical studies and clinical studies employing radio-labelled dalcetrapib that the thioester was not detectable in plasma in vivo $[15,19]$.

The thiol species is not stable in its reduced form and can dimerize or form heteromers with other molecules containing thiol groups. Both the thiol and dimeric forms might be absorbed. Although hydrolysis of the thioester produces the reduced thiol, in many solutions, and in particular under conditions of higher $\mathrm{pH}$ typical of intestinal fluid, the oxidized dimeric form was the predominant species detected. The dimer has adequate solubility in gastrointestinal fluids, and could have even greater solubility in a lipid-rich postprandial environment. It is anticipated that the thiol and dimer are in dynamic equilibrium and it has been demonstrated that the thiol, rather than the dimer, forms the majority of the 'active form' in plasma. Hence, while absorption of the dimer and subsequent reduction cannot be excluded, this suggests that the thiol may be directly absorbed under physiological conditions. Nevertheless, based on the currently available data the precise molecular species absorbed cannot be identified. These dimeric and heteromeric forms co-exist in a dynamic oxidation-reduction equilibrium with the thiol. Overall, the bioavailability of the thiol is low, and limited by extensive first-pass metabolism [16].

The primary metabolic route of dalcetrapib is outlined in Fig. 1. The thiol may be conjugated to endogenous low molecular weight thiols and high molecular weight thiolcontaining peptides and proteins to form mixed disulfides and dalcetrapib-SS-Prot. In addition, the thiol can be transformed to a dimer. These forms are considered to be in oxidation-reduction equilibrium with each other via the thiol. The thiol is further metabolized to the S-glucuronide conjugate and the S-methyl conjugate. These metabolites 


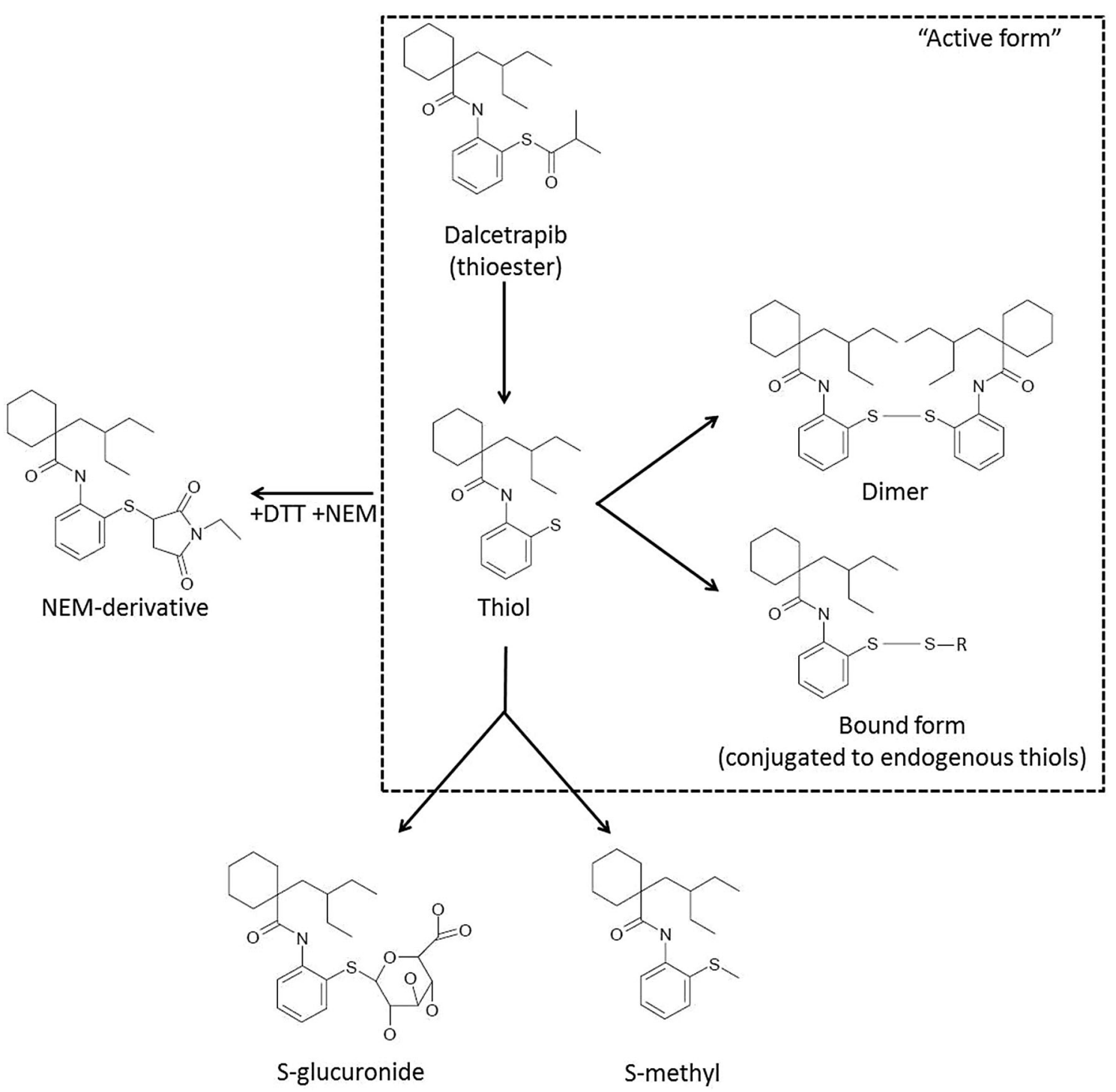

Fig. 1 Primary metabolic route of dalcetrapib. DTT dithiothreitol, NEM N-ethylmaleimide

are further metabolized to polar metabolites by hydroxylation of the substituted cyclohexyl group [18].

The primary route of elimination of the dalcetrapib-thiol is metabolism (Fig. 1). Following the administration of a single $600 \mathrm{mg}$ oral dose of ${ }^{14} \mathrm{C}$-labeled dalcetrapib, onethird and two-thirds of the total radioactivity were recovered in the urine and feces, respectively. Only a small proportion $(<1 \%)$ of the administered dose was excreted unchanged in the urine [16].

\section{Pharmacokinetics}

Following administration of dalcetrapib at once daily doses of between 300 and $1200 \mathrm{mg}$ in Caucasian subjects, the maximum concentration $\left(C_{\max }\right)$ was observed at a median time of 3-4 h post-dose (range 1-8 h), after which a biphasic elimination was observed with an average terminal $t_{1 / 2}$ of approximately $31 \mathrm{~h}$. The accumulation for the $C_{\text {max }}$ and area under the concentration-time curve (AUC) of dalcetrapib at steady state was approximately 23 and $17 \%$, respectively, and the pharmacokinetics of dalcetrapib appeared to be dose proportional over the dose range of 300-900 mg/day.

Food increases dalcetrapib active form bioavailability. The size of a meal, and time of dosing relative to a meal, influences the size of the food effect. Peak and total dalcetrapib active form exposures were higher when taken with a high-fat, high-calorie meal than when taken with a lighter meal, and when taken after a meal than when taken $30 \mathrm{~min}$ before a meal [20]. The average oral bioavailability of dalcetrapib active form among Japanese participants in 
studies conducted in Japan appears to be higher than corresponding values from studies conducted in the US, Europe, or Australasia [19].

Compared with the CETPi anacetrapib, there is no evidence of increased residence time of dalcetrapib in adipose tissue. Anacetrapib showed a long $t_{1 / 2}$ in clinical studies, and in some patients, detectable plasma concentrations were observed up to 4 years after the end of treatment [21]. Population pharmacokinetic modeling of anacetrapib indicated a deep tissue compartment consistent with accumulation in adipose tissue, which was confirmed with human tissue biopsy [22, 23]. Preliminary modeling of dalcetrapib concentrations obtained in nine clinical studies ranging from phase I to phase III (8466 samples from 2861 subjects) showed that a two-compartment model provided a good fit to the data, and the fit was not improved by adding a third compartment to simulate an adipose tissue depot (unpublished data).

\section{Pharmacodynamics}

\subsection{Safety}

A comprehensive review of the pharmacology of dalcetrapib is beyond scope of this article, which is focused on key primary pharmacology variables that provide insight into exposure versus response relationships, as seen in the dal-OUTCOMES study with broad safety, as well as the other clinical studies. The clinical program for dalcetrapib included over 25,000 patients and volunteers. Over 13,000 patients have taken dalcetrapib, of whom more than 7000 took a dose of $600 \mathrm{mg} /$ day for an average of 31 months. The safety profile of dalcetrapib is thus well-characterized, as best represented by the review of the phase II studies and the large CV endpoint study, dal-OUTCOMES $[11,24,25]$. There were no drug- or dose-related trends in heart rate, body weight, electrocardiogram, or other physical examination changes noted. The overall proportion of patients experiencing serious adverse events (AEs) was similar between dalcetrapib and placebo. More patients stopped treatment due to AEs related to diarrhea in the dalcetrapib group (1.4\%) than in the placebo group $(0.3 \%)$. [11]

\subsection{Efficacy}

Dalcetrapib is a thioester which is rapidly hydrolyzed to a thiol in biological media. The thiol is the pharmacologically active form which interacts with CETP by formation of a disulfide bond with the cysteine at residue 13 of CETP, leading to a change in CETP conformation [26]. The dalcetrapib-induced conformational change leads to a decrease in heterotypic transfer of neutral lipids (i.e., from HDL to LDL/VLDL) without impairment of homotypic transfer of CE (i.e., from HDL3 to HDL2) or CETP-dependent pre- $\beta$-HDL formation. In vivo pharmacology studies in rodent, leporid, and primate models demonstrate that dalcetrapib dosing produces increases in HDL-C and improvements in other atherogenic indices, greater cholesterol efflux from macrophages, increased RCT, and attenuation of atherosclerosis in CETP-expressing species. Overall, these results are consistent with the expectation that modulation of CETP activity by dalcetrapib treatment will have anti-atherogenic effects in humans. Recent electron microscopy binding studies also support the weaker binding affinity of dalcetrapib for HDL, and parallel the findings of earlier biochemical studies [27]. Despite the greater efficacy in inhibiting CETP, the increased HDL and reduced LDL demonstrated in the CV endpoint studies of other inhibitors of CETP have not led to new therapies [28].

Decreases in the mean CETP activity were dose dependent. For the $600 \mathrm{mg}$ dose the mean decrease in CETP activity ranged between -30.3 and $-31.6 \%$. For the $600 \mathrm{mg}$ groups in patients with $\mathrm{CHD}$ or $\mathrm{CHD}$ risk equivalent and average or low HDL-C, changes in CETP activity in different phase IIa studies ranged from an increase of $8.8 \%$ to a decrease of $-17.3 \%$. The greatest inhibition in CETP activity, -53.5 and $-56.5 \%$, was demonstrated following the administration of dalcetrapib in combination with atorvastatin for 24 and 48 weeks, respectively [19, 25, 29].

Since the response to dalcetrapib appeared to be highest in the subset of patients with AA genotype at rs1967309, the pharmacokinetics of dalcetrapib for the various genotypes were evaluated as a possible explanation. The population pharmacokinetic model for dalcetrapib was used to estimate drug clearance for individual subjects $(n=2861)$ from the dal-OUTCOMES study and these clearance values are plotted by genotype in Fig. 2. No difference was observed between genotypes, suggesting that greater response to dalcetrapib associated with the AA genotype was not due to higher drug exposure in these patients. Ninety-five percent of patients were taking statins (HMGCoA reductase inhibitors) in this study (consistent with expected clinical exposure), so a pharmacokinetic interaction by genotype in patients not concurrently taking statins is not available. There were also no differences in HDL-C between genotype in the dal-OUTCOMES study [12].

\subsection{Additional Pharmacodynamic Effects}

During the development of the drug, human biomaterials were utilized to investigate the pharmacodynamic effects of dalcetrapib in vitro. A significant effort was commenced 
Fig. 2 Model-predicted dalcetrapib clearance for individual subjects by genotype.

The horizontal line represents the overall median of 606 subjects from the dal-

OUTCOMES study $(\mathrm{AA}=99$ subjects, $A G=283$ subjects, $\mathrm{GG}=224$ subjects) [data derived from dal-Outcomes $[11,12]$ but were not part of the published analysis]. $C L$ clearance

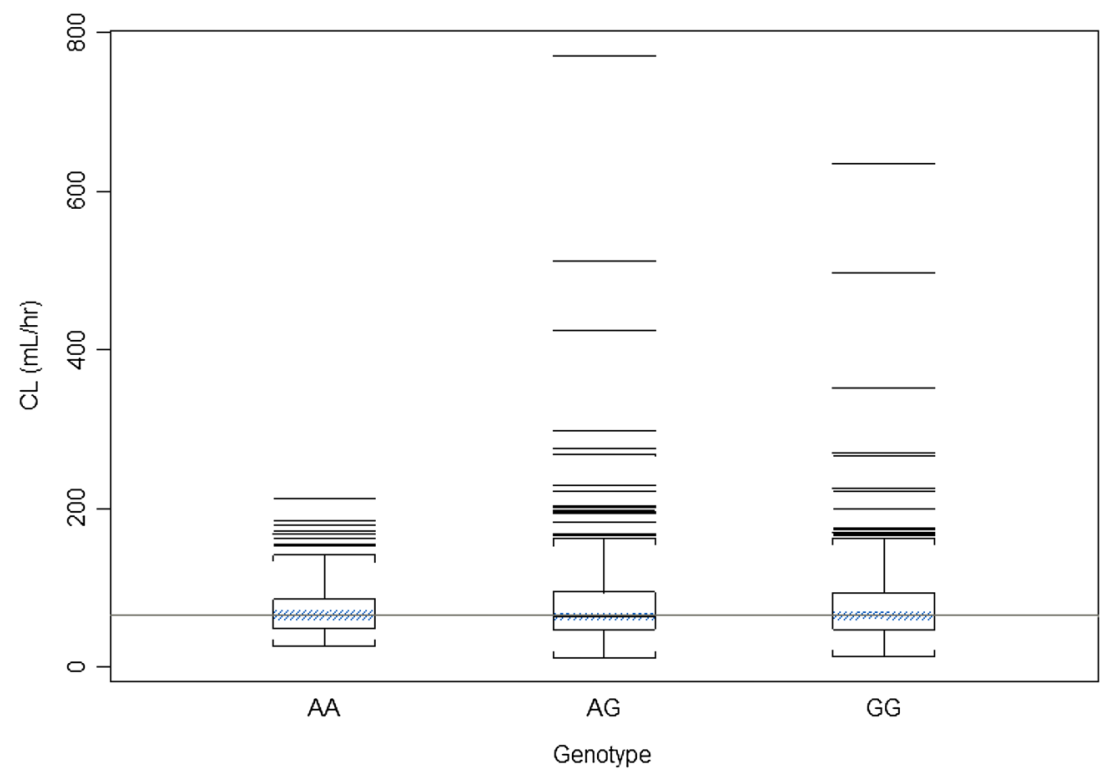

to assess whether there were any pharmacodynamic effects unrelated to CETP activity, but none were identified despite significant exploration [30]. These studies used human biomaterials to test the affinity of dalcetrapib thioester for a variety of receptors and the inhibitory potency against a number of enzymes. The effect of dalcetrapib thioester on aldosterone secretion and expression of genes involved in aldosterone and cortisol synthesis was also explored in cultured human adrenocortical carcinoma cells. To assess the potential for dalcetrapib dosing to prolong the QT interval in vivo, the effect of dalcetrapib thioester on the hERG (human ether-à-go-go related gene) potassium current was measured in cultured cells. No clinically relevant prolongation of QT interval was observed in clinical studies [31].

\section{Drug-Drug Interactions}

\subsection{Interactions with Statins and Ezetimibe}

In the statin drug-drug interaction studies, the plasma exposure of dalcetrapib appeared to be reduced when administered in combination with simvastatin, atorvastatin, and rosuvastatin, but no changes in the exposures of simvastatin, atorvastatin, or rosuvastatin or any measured active metabolites were observed that are considered to be clinically relevant taking into account the relative activity of the analytes or their variability in exposure [32-34]. Although the $C_{\max }$ and AUC from time zero to $24 \mathrm{~h}$ $\left(\mathrm{AUC}_{24}\right)$ values of pravastatin were reduced following coadministration of pravastatin with dalcetrapib, a decrease of approximately $25 \%$ did not result in an apparent loss of the LDL-C-lowering effect in plasma, which suggests that the reduction in plasma exposure may not be clinically relevant. The reduction in the plasma exposure of dalcetrapib when combined with rosuvastatin, atorvastatin, simvastatin, and pravastatin did not result in any apparent relevant change in its effects on CETP activity or HDL-C [30-32]. Results from the ezetimibe drug-drug interaction study demonstrated that plasma concentrations of dalcetrapib were unchanged following repeat co-administration of dalcetrapib $900 \mathrm{mg}$ and ezetimibe $10 \mathrm{mg}$ compared with dalcetrapib alone [35]. The calculated plasma exposure to conjugated ezetimibe was $22.5 \%$ lower after coadministration with dalcetrapib, while unconjugated ezetimibe showed a small increase in $C_{\max }$ only. There was no effect on the measured pharmacodynamic parameters as a result of the decreased ezetimibe exposure. Differential distribution into LDL, VLDL/chylomicrons and HDL may explain observed drug-drug interactions between dalcetrapib and lipid-modifying agents (i.e., a reduction in plasma exposure but no apparent decrease in dalcetrapib treatment effects from statins) [36, 37].

In the 15,871-patient dal-OUTCOMES study, all patients had to be following individualized, evidence-based programs for lowering their LDL-C levels by means of statin therapy and diet, with a target LDL-C level of $100 \mathrm{mg} / \mathrm{dL}$ or lower, and preferably $70 \mathrm{mg} / \mathrm{dL}$ or lower. However, no specific statin agent or dose was specified. Most patients $(98 \%$ in the placebo group and $97 \%$ in the dalcetrapib group) were treated with statins. No apparent effects of dalcetrapib on LDL-C concentration were seen when added onto an established lipid-modifying treatment regimen in patient studies (either defined statin or 'standard of care'). There was no increase in the incidence of statin- 
related AEs such as hepatic transaminase elevation, myopathy, or rhabdomyolysis in the dal-OUTCOMES study, and the incidence of these AEs is expected to be similar to that of statin-treated patients [11, 24, 25].

\subsection{Interactions Other Than Statins}

Co-administration of clinical doses of orlistat with dalcetrapib significantly reduced the dalcetrapib active form peak and total exposure by over $50 \%$, as well as reducing CETP activity [37]. The interaction is postulated to be the consequence of inhibition of de-esterification in the gastrointestinal tract (i.e., reduced oral bioavailability).

Results from the drug-drug interaction studies indicate that dalcetrapib has no major effect on cytochrome $\mathrm{P} 450$ (CYP) 1A2, 2C8, 2C9, 2C19, 2D6, or 3A4. Exposure of the active form decreased or remained unchanged with coadministration of dalcetrapib (900 and $600 \mathrm{mg}$ ) and ketoconazole, indicating that the active form is not a substrate for CYP3A4 [38]. An apparent in vitro CYP inhibitory effect was studied in subsequent clinical trials, and at $900 \mathrm{mg}$ no clinically relevant effect was demonstrated [39].

The effect of multiple-dose administration of dalcetrapib when given concomitantly with a combined oral contraceptive pill, Microgynon ${ }^{\circledR}-30$, was investigated in healthy female subjects and no significant pharmacokinetic or pharmacodynamic effects were identified [40]. In healthy subjects, there was no clinically relevant pharmacokinetic interaction between single doses of dalcetrapib $900 \mathrm{mg}$ and digoxin $0.5 \mathrm{mg}$ [41]. Additionally, there was no clinically relevant pharmacokinetic interaction between dalcetrapib and probenecid, an inhibitor of drug transporters and uridine 5'-diphospho-glucuronosyltransferase (UGT) enzymes [42].

\subsection{Clinical Significance of Drug-Drug Interactions}

Drug-drug interactions are of paramount concern to any prescribing physician, and the lack of clinically significant interactions between dalcetrapib and most drugs commonly prescribed to $\mathrm{CV}$ patients is reassuring. In the dal-OUTCOMES study, $97 \%$ of the patients were also receiving aspirin, $89 \%$ received concomitant clopidogrel, ticlopidine, or prasugrel, $87 \%$ also took a $\beta$-blocker, and $79 \%$ also received an ACE inhibitor or angiotensin receptor blocker with no evidence of clinically relevant drug interactions. As predicted by the lack of effect in the drug-drug interaction studies, the results do not suggest any clinically relevant interactions with drugs other than the lipase inhibitor orlistat [10, 43, 44].

\section{Pharmacokinetics in Special Populations}

Mild hepatic impairment has little effect on the pharmacokinetics of dalcetrapib and metabolites, while moderate hepatic impairment altered dalcetrapib and metabolite pharmacokinetics. Mean total and peak exposure of dalcetrapib was 34 and 38\% higher among subjects with moderate hepatic impairment, respectively, compared with that of age-, weight-, and sex-matched controls. However, no dose adjustment is necessary in patients with mild or moderate hepatic impairment (i.e., Child-Pugh A or B). Dalcetrapib has not been studied in patients with severe hepatic impairment (i.e., Child-Pugh C) [45].

Dalcetrapib-thiol exposure was higher among subjects with moderate and severe renal impairment than in individuals with normal renal function. Mean total exposure of dalcetrapib-thiol was 62 and $81 \%$ higher among subjects with moderate or severe renal impairment, respectively, than in age-, weight-, and sex-matched controls. Dalcetrapib-thiol exposure also appeared to be slightly higher in the group of subjects with mild renal impairment than in the control group with normal renal function. However, no dose adjustment is necessary in patients with mild, moderate, or severe renal impairment (i.e., creatinine clearance $\left[\mathrm{CL}_{\mathrm{CR}}\right] \geq 15 \mathrm{~mL} / \mathrm{min}$ ). Dalcetrapib has not been studied in patients with end-stage renal disease [45].

There are limited clinical implications of dalcetrapib use in any population. The long-term clinical studies included patients with mild to moderate hepatic and renal dysfunction, and there was no increase in adverse effects. In the 15,871-patient dal-OUTCOMES trial, the mean changes in safety laboratory values were similar between the treatment arms for chemistry and hematology variables. The incidence of laboratory abnormalities was also similar between arms. Dalcetrapib had no significant effect on measures of hepatic or renal function or on creatine kinase levels and no effect on fasting plasma glucose or glycated hemoglobin levels [11].

\section{Conclusions}

Dalcetrapib is under investigation to be the first CETPi approved for the reduction of CV events. While a lesspotent inhibitor of CETP, only dalcetrapib is under consideration for treatment of patients with a singular polymorphism of the ADCY9 gene at chromosome 16. In a subgroup analysis of several prospective studies, this population, which had a background of excellent statin therapy, benefited from dalcetrapib with a significant reduction in $\mathrm{CV}$ events over a period of less than 3 years. 
Chemically, dalcetrapib is a thioester prodrug, which is hydrolyzed to generate a pharmacologically active thiol. The oral bioavailability is low and limited due to extensive first-pass metabolism. Food increases the 'active form' exposure, and the size of the meal influences the size of the food effect. Clearance occurs by metabolism via glucuronic acid conjugation and methylation of the sulfhydryl group. Exposure appears to be both dose- and time-dependent, and is moderately higher in patients with hepatic or renal impairment. Overall, the drug appears to be well-tolerated in over 13,000 patients and volunteers exposed to dalcetrapib, with only an increase in diarrhea reported as a significant adverse effect. Specific drug-drug interaction studies had no issues with any drugs commonly prescribed to cardiac patients, and the experience in a large CV endpoint study did not indicate any issues. Population pharmacokinetic analysis based on the large patient population also showed no significant effect based on genotype.

Acknowledgments The authors would like to thank Nancy Dolphin, Lauren Heinonen, and Ryan Black for providing assistance with figures and text.

\section{Compliance with Ethical Standards}

Funding The studies discussed in this review were funded by Japan Tobacco (JTT), Roche, and DalCor Pharma UK Ltd.

Conflict of interest Donald Black and Therese Heinonen are employees of DalCor Pharma UK Ltd., Stockport, UK. Darren Bentley is an employee of Roche, Switzerland. Sunny Chapel, Jongtae Lee, and Emily Briggs are employees of A2PG, which is a consulting company to DalCor Pharma.

Open Access This article is distributed under the terms of the Creative Commons Attribution-NonCommercial 4.0 International License (http://creativecommons.org/licenses/by-nc/4.0/), which permits any noncommercial use, distribution, and reproduction in any medium, provided you give appropriate credit to the original author(s) and the source, provide a link to the Creative Commons license, and indicate if changes were made.

\section{References}

1. Chapman MJ, Le Goff W, Guerin M, Kontush A. Cholesteryl ester transfer protein: at the heart of the action of lipid-modulating therapy with statins, fibrates, niacin, and cholesteryl ester transfer protein inhibitors. Eur Heart J. 2010;31(2):149-64.

2. Tall A. Functions of cholesterol ester transfer protein and relationship to coronary artery disease risk. J Clin Lipidol. 2010;4(5):389-93.

3. Charles MA, Kane JP. New molecular insights into CETP structure and function: a review. J Lipid Res. 2012;53(8):1451-8.

4. Khera A, Rader D. Future therapeutic directions in reverse cholesterol transport. Curr Atheroscler Rep. 2010;12(1):73-81.

5. Annema W, Willemsen H, deBoer J, Dikkers A, van der Giet M, Nieuwland W, et al. HDL function is impaired in acute myocardial infarction independent of plasma HDL cholesterol levels. J Clin Lipid. 2016;10:1318-28.
6. Barter P, Caulfield M, Eriksson M, Grundy S, Kastelien J, Komajda M, et al. Effects of torcetrapib in patients at high risk for coronary events. N Engl J Med. 2007;357(21):2109-22.

7. Stroes ES, Kastelein JJ, Benardeau A, Kuhlmann O, Blum D, Campos LA, et al. Dalcetrapib: no off-target toxicity on blood pressure or on genes related to the renin-angiotensin-aldosterone system in rats. Br J Pharmacol. 2009;158(7):1763-70.

8. HPS 3/TIMIT55-REVEAL Collaborative Group. Effects of anacetrapib in patients with atherosclerotic vascular disease. N Engl J Med. 2017;377:1217-27.

9. Lincoff A, Nicholls S, Riesmeyer J, Barter P, Brewer H, Fox K, et al. Evacetrapib and cardiovascular outcomes in high-risk vascular disease. N Engl J Med. 2017;376:1933-42.

10. Okamoto H, Yonemori F, Wakitani K, Minowa T, Maeda K, Shinkai $\mathrm{H}$. A cholesteryl ester transfer protein inhibitor attenuates atherosclerosis in rabbits. Nature. 2000;406(6792):203-7.

11. Schwartz GG, Olsson AG, Abt M, Ballantyne CM, Barter PJ, Brumm $\mathrm{J}$, et al. Effects of dalcetrapib in patients with a recent acute coronary syndrome. N Engl J Med. 2012;367(22):2089-99.

12. Tardif JC, Rheaume E, Lemieux Perreault LP, Gregoire JC, Feroz Zada Y, Asselin G, et al. Pharmacogenomic determinants of the cardiovascular effects of dalcetrapib. Circ Cardiovasc Genet. 2015;8(2):372-82.

13. Tardif JC, Rhainds D, Brodeur M, Feroz Zada Y, Fouodjio R, Provost S, et al. Genotype-dependent effects of dalcetrapib on cholesterol efflux and inflammation: concordance with clinical outcomes. Circ Cardiovasc Genet. 2016;9(4):340-8.

14. Shinkai H, Maeda K, Yamasaki T, Okamoto H, Uchida I. bis(2(Acylamino)phenyl) disulfides, 2-(acylamino)benzenethiols, and S-(2-(acylamino)phenyl) alkanethioates as novel inhibitors of cholesteryl ester transfer protein. J Med Chem. 2000;43(19):3566-72.

15. Gross G, Tardio J, Kuhlmann O. Solubility and stability of dalcetrapib in vehicles and biological media. Int $\mathrm{J}$ Pharm. 2012;437(1-2):103-9.

16. Bentley DJ, Phelan M, Derks M, Ishikawa T, Takubo H, Kuhlmann $\mathrm{O}$, et al. Absorption and disposition of dalcetrapib, a thioester prodrug of a thiol, in humans. TBD. 2017 (unpublished data).

17. Husser C, Pähler A, Seymour M, Kuhlmann O, Schadt S, Zell M. Profiling of dalcetrapib metabolites in human plasma by accelerator mass spectrometry combined with structure elucidation by high resolution mass spectrometry. J Pharm Biomed Anal. 2018;152:143-54.

18. Heinig K, Bucheli F, Kuhlmann O, Zell M, Pahler A, Zwanziger E, et al. Determination of dalcetrapib by liquid chromatographytandem mass spectrometry. J Pharm Biomed Anal. 2012;66:314-24

19. Derks M, Anzures-Cabreba J, Turnbull L, Phelan M. Safety, tolerability and pharmacokinetics of dalcetrapib following single and multiple ascending doses in healthy subjects - a randomized, double-blind, placebo-controlled, phase I study. Clin Drug Investig. 2011;31(5):325-35.

20. Derks M, Kawamura H, Abt M, Meneses-Lorente G, Phelan M, Ishikawa T. Effects of food intake on the pharmacokinetic properties of dalcetrapib: findings from three phase I, single-dose crossover studies in healthy volunteers. Clin Ther. 2011;33(6):754-65.

21. Gotto A, Cannon C, Li X, Vaidya S, Kher U, Brinton EA, et al. Evaluation of lipids, drug concentration, and safety parameters following cessation of treatment with the cholesteryl ester transfer protein inhibitor anacetrapib in patients with or at high risk for coronary heart disease. Am J Cardiol. 2014;113(1):76-83.

22. Small DS, Ke AB, Hall SD, Mantlo N, Rotelli M, Friedrich S. Assessment of the persistence of anacetrapib and evacetrapib 
concentrations using two pharmacokinetic modeling approaches. J Clin Pharmacol. 2015;55(7):757-67.

23. Krishna R, Gheyas F, Liu Y, Hagen DR, Walker B, Chawla A, et al. Chronic administration of anacetrapib is associated with accumulation in adipose and slow elimination. Clin Pharmacol Ther. 2017;102(5):832-40.

24. Stein EA, Stroes ES, Steiner G, Buckley BM, Capponi AM, Burgess $\mathrm{T}$, et al. Safety and tolerability of dalcetrapib. Am J Cardiol. 2009;104(1):82-91.

25. Stein EA, Roth EM, Rhyne JM, Burgess T, Kallend D, Robinson JG. Safety and tolerability of dalcetrapib (RO4607381/JTT-705): results from a 48-week trial. Eur Heart J. 2010;31(4):480-8.

26. Maugeais C, Perez A, von der Mark E, Magg C, Pflieger P, Niesor EJ. Evidence for a role of CETP in HDL remodeling and cholesterol efflux: role of cysteine 13 of CETP. Biochim Biophys Acta. 2013;1831(11):1644-50.

27. Zhang M, Lei D, Peng B, Yang M, Zhang L, Charles MA, et al. Assessing the mechanisms of cholesteryl ester transfer protein inhibitors. Biochem Biophys Acta. 2017;1862(12):1606-17.

28. Ferri N, Corsini A, Sirtori CR, Ruscica M. Present therapeutic role of cholesteryl ester transfer protein inhibitors. Pharmacol Res. 2018;26(128):29-41.

29. Cunningham D, Lin W, Hoth LR, Danley DE, Ruggeri RB, Geoghegan KF, et al. Biophysical and biochemical approach to locating an inhibitor binding site on cholesteryl ester transfer protein. Bioconjug Chem. 2008;19(8):1604-13.

30. Ranalletta M, Bierilo KK, Chen Y, Milot D, Chen Q, Tung E, et al. Biochemical characterization of cholesteryl ester transfer protein inhibitors. J Lipid Res. 2010;51(9):2739-52.

31. Derks M, Abt M, Mwangi A, Meneses-Lorente G. Lack of effect of dalcetrapib on QT interval in healthy subjects following multiple dosing. Eur J Clin Pharmacol. 2010;66(8):775-83.

32. Derks M, Abt M, Parr G, Meneses-Lorente G, Young AM, Phelan M. No clinically relevant drug-drug interactions when dalcetrapib is co-administered with atorvastatin. Expert Opin Investig Drugs. 2010;19(10):1135-45.

33. Derks M, Abt M, Phelan M, Turnbull L, Meneses-Lorente G, Bech N, et al. Coadministration of dalcetrapib with pravastatin, rosuvastatin, or simvastatin: no clinically relevant drug-drug interactions. J Clin Pharmacol. 2010;50(10):1188-201.

34. Kuivenhoven JA, de Grooth GJ, Kawamura H, Klerkx AH, Wilhelm F, Trip MD, et al. Effectiveness of inhibition of cholesteryl ester transfer protein by JTT-705 in combination with pravastatin in type II dyslipidemia. Am J Cardiol. 2005;95(9):1085-8.

35. Derks M, Abt M, Phelan M. Lack of clinically relevant drug-drug interactions when dalcetrapib is co-administered with ezetimibe. Br J Clin Pharmacol. 2010;70(6):825-33.
36. Ballantyne CM, Miller M, Niesor EJ, Burgess T, Kallend D, Stein EA. Effect of dalcetrapib plus pravastatin on lipoprotein metabolism and high-density lipoprotein composition and function in dyslipidemic patients: results of a phase IIb dose-ranging study. Am Heart J. 2012;163(3):515-21 (521.e1-3).

37. Bentley D, Young AM, Rowell L, Gross G, Tardio J, Carlile D. Evidence of a drug-drug interaction linked to inhibition of ester hydrolysis by orlistat. J Cardiovasc Pharmacol. 2012;60(4):390-6.

38. Derks M, Fowler S, Kuhlmann O. A single-center, open-label, one-sequence study of dalcetrapib coadministered with ketoconazole, and an in vitro study of the S-methyl metabolite of dalcetrapib. Clin Ther. 2009;31(3):586-99.

39. Derks M, Fowler S, Kuhlmann O. In vitro and in vivo assessment of the effect of dalcetrapib on a panel of CYP substrates. Curr Med Res Opin. 2009;25(4):891-902.

40. Young AM, Anzures-Cabrera J, Derks M. No clinically relevant drug-drug interactions when dalcetrapib is co-administered with a monophasic oral contraceptive (Microgynon ${ }^{\circledR}$ 30). Int J Clin Pharmacol Ther. 2012;50(4):248-56.

41. Young AM, Anzures-Cabrera J, Bentley D, Carlile D. Dalcetrapib, a cholesteryl ester transfer protein modulator, has no apparent effect on digoxin exposure. British Pharmacological Society Winter Meeting; 2011; London, UK, 13th-15th December 2011.

42. Aceves Baldo P, Anzures-Cabrera J, Bentley D. In vivo evaluation of drug-drug interactions linked to UGT inhibition: the effect of probenecid on dalcetrapib pharmacokinetics. Int J Clin Pharmacol Ther. 2013;51(3):215-8.

43. Schwartz GG, Olsson AG, Ballantyne C, Barter P, Holme I, Kallend D, et al. Rationale and design of the dal-Outcomes trial: efficacy and safety of dalcetrapib in patients with recent acute coronary syndrome. Am Heart J. 2009;158(6):896-901.

44. Maugeais C, von der Mark E, Niesor EJ, Heinig K, MenesesLorente G, Derks M, et al. Plasma lipoprotein fraction distribution of the CETP modulator dalcetrapib and the relationship with pharmacodynamic effects. XVIth International Symposium on Athersclerosis-HDL Satellite Symposium; 2012; Cairns, Australia, 30th March 2012-1st April 2012.

45. Phelan M, Anzures-Cabrera J, Carlile DJ, Rowell L, Kuhlmann $\mathrm{O}$, Arold G, et al. Effect of hepatic and renal impairment on the pharmacokinetics of dalcetrapib: altered distribution of the active thiol. Clin Pharmacokinet. 2013;52(4):255-65.

46. DalCor Pharmaceuticals. Effect of dalcetrapib vs placebo on CV risk in a genetically defined population with a recent ACS (dalGenE) [ClinicalTrials.gov identifier NCT02525939]. US National Institutes of Health, ClinicalTrials.gov. https://www.clinicaltrials. gov. Accessed 5 Apr 2018. 